\title{
BIOSCANNER-A DEVICE RECORDING AUTOMATICALLY THE COURSES OF MICROBIAL GROWTH THROUGH SCANNING PROCEDURE WITH TWENTY-ONE CHANNELS ${ }^{1}$
}

\author{
HIROSHI KURAISHI, TOSHIHIRO MIKAMI*, KAZUKO TASHIRO \\ AND TEIJIRO UEMURA \\ The Institute of Applied Microbiology, University of Tokyo, \\ Bunkyo-ku, Tokyo \\ and \\ *Otake Works Ltd., 2-7-7, Nishi-azabu, Minato-ku, Tokyo
}

(Received June 23, 1967)

\begin{abstract}
An apparatus "Bioscanner", which is able to record automatically growth curves of 21 samples as a lot, was constructed. This has a scanning device with a shiftable light source connected with a photocell. The cultivation is carried out in L-form tubes fixed on a holder, which is placed in a rolling water bath. Growth curves of 21 specimens are recorded on an endless chart. A linear relationship between transmission value and the logarithm of cell density can be hold at the range of $15 \%$ to $100 \%$ transmission.

Some examples of characteristic growth curves are presented employing test organisms of different properties.
\end{abstract}

Growth of unicellular microorganisms such as yeast and bacteria is photometrically traced at intervals. This burdens microbiologists with a laborious and rather hazardous work. A "Biophotometer" (Jouan Co., Paris) enables us to measure the growth automatically, but this apparatus permits to set only six samples as a lot, so that it is not well applicable to research works treating many samples at the same time. The "Biophotometer" is characterized by successive shifts of six photocell circuits equipped on six sample tubes against a fixed lamp placed in the center. By contrast, a scanning device capable of shifting a set of a light source and a photocell against a number of sample tubes seems to be much easier to scan larger number of samples. The present report describes an apparatus constructed along this line employing improved recording system, which automatically depicts growth curves of at least twenty-one samples as a lot.

1 This investigation was supported in part by a grant from the Ministry of Education. 


\section{EXPERIMENTALS}

Scanning apparatus. This apparatus, which is tentatively named "Bioscanner", is mainly constituted of a water bath for cultivation, a shifting light source and a photocell, a chain device for scanning, and an automatic

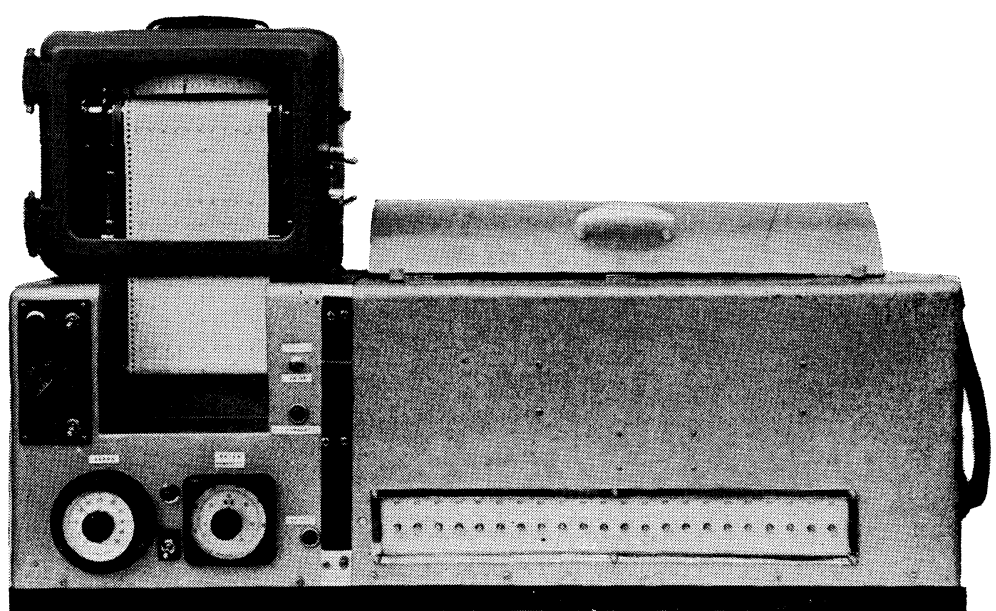

Fig. 1. Whole view of Bioscanner.

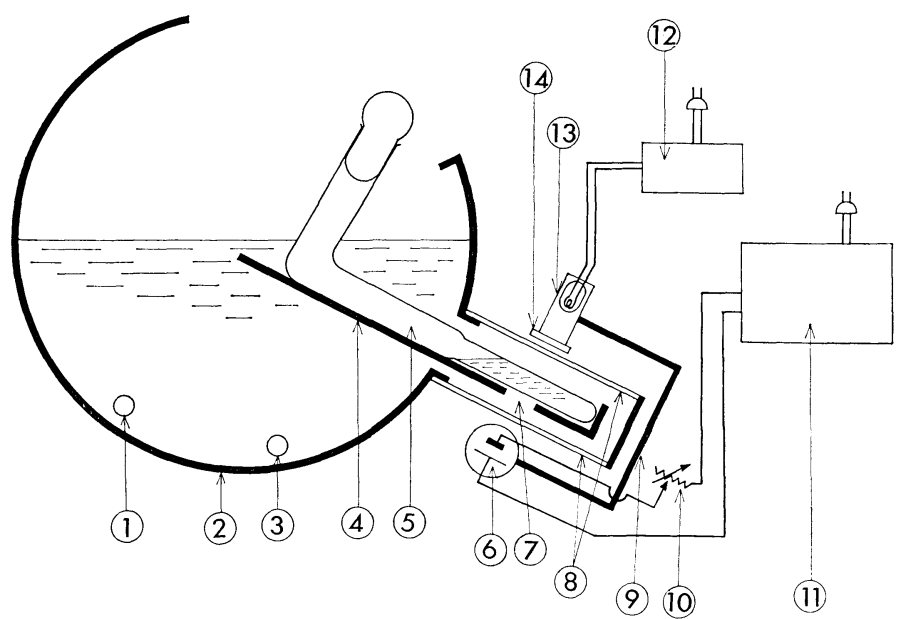

Fig. 2. Schematic diagram showing a cross view of Bioscanner.

1. Pipe heater

2. Water bath

3. Thermistor

4. Holder of L-tubes

5. L-tube

6. Photocell

7. Light-transmission hole
8. Glass windows on projecting chamber

9. Connector between light source and photocell

10. Variable resistor

11. Recorder

12. Stabilizer

13. Light sot $\mathbf{r}$ ze

14. Glass filter 
recorder (Figs. 1 and 2). L-form tubes as shown in Fig. 2, the basal part of which is flat and square-shaped in cross section, are used as containers of culture liquid and fitted on a holder of the apparatus.

The water bath equipped in the apparatus is a horizontal cylinder with $20 \mathrm{~cm}$ in diameter and $50 \mathrm{~cm}$ in length. The bath possesses a projecting chamber (see Fig. 2), whose upper and lower sides are equipped with glass windows for turbidity scanning. An agitation of water in bath and media in the L-form tubes is carried out by rolling back and forth the entire water bath with an amplitude angle of $7^{\circ}$ (20 times/min). The agitation of water is effected by water flows into and out of the projecting chamber of the bath by the rolling movement.

As described above, the basal part of the $L$-form tube is deformed into

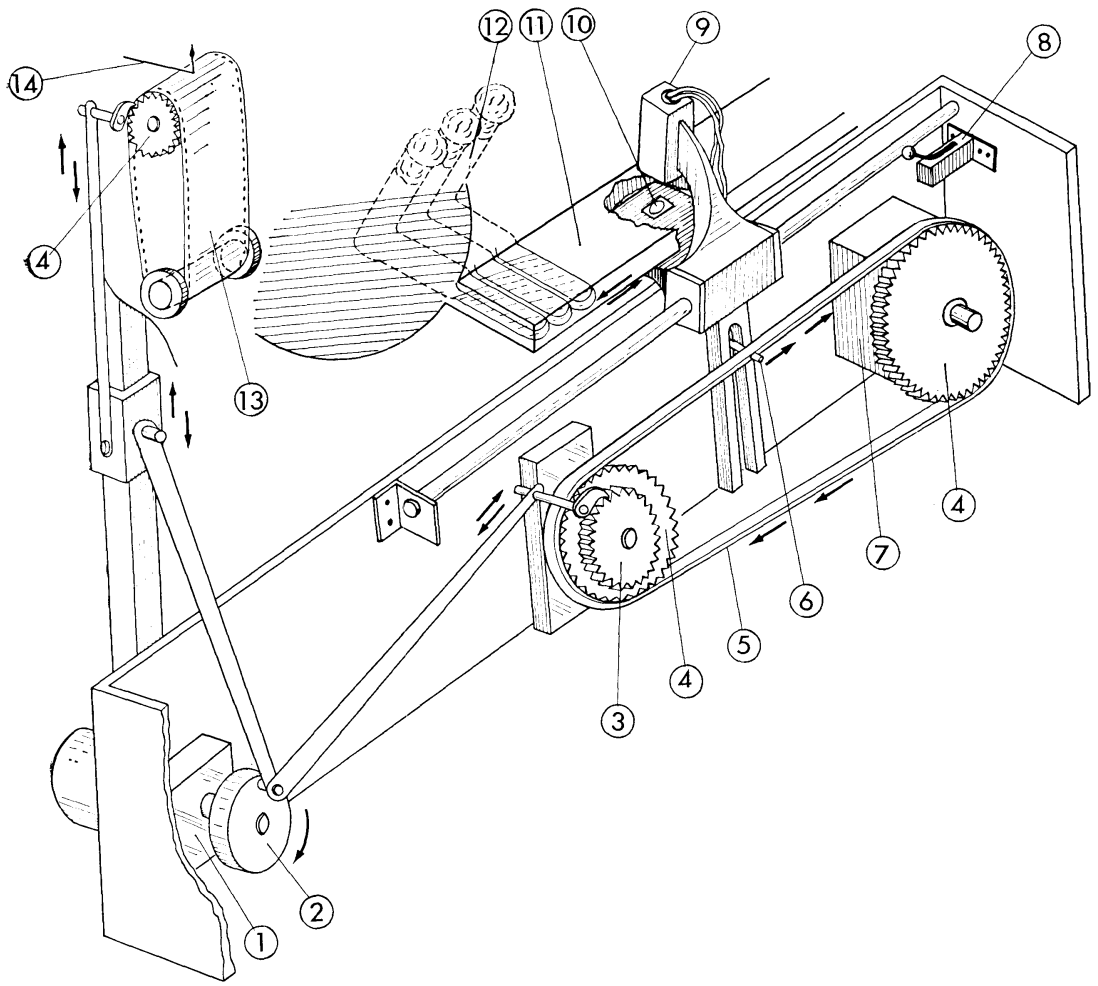

Fig. 3. Schematic representation of a chain movement device in Bioscanner.

1. Motor A

2. Cam

3. Ratchets

4. Sprockets

5. Chain

6. Pin fixed on chain

7. Motor B
8. Microswitch

9. Light source

10. Photocell

11. Projecting chamber of water bath

12. L-form tube

13. Looped chart

14. Needle of ammeter 
a flat shape of $10 \mathrm{~mm}$ in thickness and the volume of the square portion is about $9.3 \mathrm{ml}$ (total volume of the L-form tube being about $27 \mathrm{ml}$ ). Twentyone L-form tubes are set on stoppers equipped on the holder having 21 holes for the light transmission, and, when the holder is fixed in the bath; the basal parts of the tubes are just placed in the projecting chamber of the bath. The transmission values of the tubes themselves often vary very much. Therefore, determination of the transmission value of the tubes is required, and those tubes having $\pm 2 \%$ or more deviation against the mean value should be eliminated.

Prior to the turbidity measurement, the rolling of the water bath is stopped automatically keeping its position which enables to fill up the projecting chamber with water. The light turned on during operation of scanning system passes through $660 \mathrm{~m} \mu$ glass filter and is received by a photocell through each sample tube in water. A photocell holder connecting the photocell and the light source shifts successively from one tube to the other by the action of chain movement from left to right hand side. As shown in Fig. 3, the rotary movement of a cam is transmitted by a lever to a ratchet tightly connected with a sprocket to move the chain and by another lever to a recorder to record electric response to the transmitted light. It should be mentioned that both levers have a common fulcrum on the cam. After a shift from one tube to the other, the photocell holder stands still for 11 sec just on each light-transmitting hole of the holder. This transitory stop mechanism of the photocell holder is constructed precisely so as to stop at

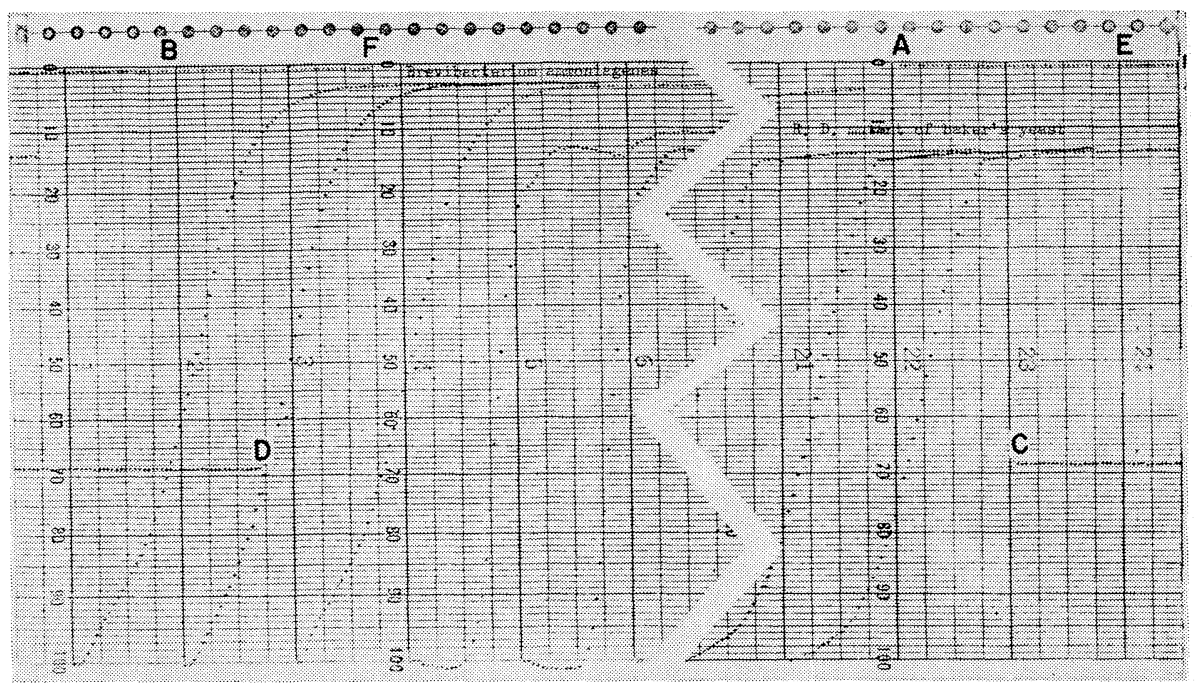

Fig. 4. A part of a recorded chart. Numbers printed on the center line show start lines of test plots (1-21) and 3 monitoring plots. A-B, C-D and E-F show the first, second and third monitoring plots, respectively. 
exactly the same position at every scanning procedures. After the measurement of the last sample, a microswitch works, and the moter A stops, while the other moter $\mathrm{B}$ begins to operate, and then the photocell and the light source returns rapidly back to the initial left hand side terminal. Then, the water bath resumes to keep its position so as to be able to measure the \% transmission and then commences rolling again. The shaking pericd is ccrtrolled by setting a timer in a range up to $60 \mathrm{~min}$. Since it takes $3.5 \mathrm{~min}$ for one measurement, if one wants to plot every ten min, for instance, the timer must be set at the scale of $6.5 \mathrm{~min}$.

The recorder consists of a moving coil ammeter and three different color-plotting systems. An endless, looped recording chart $(15.1 \mathrm{~cm}$ in w:dth) is made by pasting up both terminals of the chart cut into a lencth in $60 \mathrm{~cm}$ (Fig. 4). The endless chart moves $25 \mathrm{~mm}$ in distance for every lutings of the transmission of every tube. Consequently, the looped chart comretes its cycle after plotting 24 times. Among 24 plots depicted on the chart, the initial three plots are employed for three kinds of monitorings durirg tion. The first plot (A to B in Fig. 4) is used to monitor $0 \%$ transmissicn by adjusting manually at first the ammeter and by checking its fluctuation during operation. The second (C to D in Fig. 4) is a monitor of the circut involving the photocell and recorder. This is effected by a fixed resistor. which enables us to keep an appropriate level in transmission value. The third one ( $\mathrm{E}$ to $\mathrm{F}$ in Fig. 4) monitors whether the photocell receives extralight that possibly comes through rifts of the apparatus case. By such a monitoring device one can safely operate this apparatus by checking these plots only frequently. After every 24 plottings, the chart moves $1 \mathrm{~mm}$ each, and thus 21 growth curves and 3 monitory lines plotted at space intervals of $1 \mathrm{~mm}$ along the time axis are obtained (Fig. 4). As the inoculum size and the colouration of medium cause a change in the initial transmission value, adjustment of the initial value to the basal line is carried out by controlling manually each variable resistor placed in a circuit for each sample before the commencement of the automatic operation.

Cultures. Bacillus megaterium IAM 1166 (NRRL B-939), Escherichia coli K 12, Pseudomonas alcaligenes ATCC 12815 and baker's yeast BA-1 employed in the previous work (1) were employed as test organisms.

Media. In Table 1, compositions of media are listed. For B. megaterium, Martin and Foster's medium (2) as modified by YAMAKAWA et al. (3) was used (Medium A). Two parts of the medium described in Table 1 were sterilized separately and combined in a ratio of $1: 9$ by volume before use. Ordinary nutrient broth (Medium B) is used for cultivations of $E$. coli and $P$. alcaligenes. For yeast, pantothenate-deficient synthetic medium (Medium C) was employed.

Growth conditions. Unless otherwise noted, all growth experiments were carried out at $30^{\circ}$ in L-form tubes containing $6 \mathrm{ml}$ of media and the optical changes were recorded 4 times an hour. 
Table 1. Compositions of media.

Medium A (employed for Bacillus megaterium)

Part 1

$\mathrm{KH}_{2} \mathrm{PO}_{4}$

$\left(\mathrm{NH}_{4}\right)_{2} \mathrm{HPO}_{4}$

Distilled water up to $100 \mathrm{ml}$
Part 2

$5.0 \mathrm{~g} \quad$ Glucose

$1.0 \mathrm{~g} \quad$ L-Glutamic acid

Yeast extract (Difco)

$\mathrm{MgSO}_{4} \cdot 7 \mathrm{H}_{2} \mathrm{O}$

$\mathrm{NaCl}$

$\mathrm{CaCl}_{2} \cdot 2 \mathrm{H}_{2} \mathrm{O}$

$\mathrm{MnSO}_{4} \cdot 4 \mathrm{H}_{2} \mathrm{O}$

$\mathrm{ZnSO}_{4} \cdot 7 \mathrm{H}_{2} \mathrm{O}$

$\mathrm{CuSO}_{4} \cdot 5 \mathrm{H}_{2} \mathrm{O}$

Distilled water
$1.0 \mathrm{~g}$

$1.0 \mathrm{~g}$

$0.5 \mathrm{~g}$

$0.4 \mathrm{~g}$

$1.0 \mathrm{~g}$

$5.8 \mathrm{mg}$

$7.0 \mathrm{mg}$

$10.0 \mathrm{mg}$

$0.064 \mathrm{mg}$ to $900 \mathrm{ml}$

Medium B (employed for Escherichia coli and Pseudomonas alcaligenes)

$\begin{array}{lr}\text { Meat extract (Kyokuto) } & 10 \mathrm{~g} \\ \text { Peptone (Kyokuto) } & 10 \mathrm{~g} \\ \mathrm{NaCl} & 5 \mathrm{~g} \\ \text { Tap water } & \text { to } 1,000 \mathrm{ml} \\ & \mathrm{pH} 6.8\end{array}$

Medium C (employed for Saccharomyces cerevisiae)

Glucose

$10.0 \mathrm{~g}$

$\mathrm{Na}_{2} \mathrm{HPO}_{4}$

$2.2 \mathrm{~g}$

Citric acid

$2.0 \mathrm{~g}$

$\mathrm{KCl}$

$0.6 \mathrm{~g}$

$\mathrm{MgSO}_{4} \cdot 7 \mathrm{H}_{2} \mathrm{O}$

$0.5 \mathrm{~g}$

$\mathrm{CaCl}_{2} \cdot 6 \mathrm{H}_{2} \mathrm{O}$

$0.1 \mathrm{~g}$

$\mathrm{H}_{3} \mathrm{BO}_{3}$

$500 \mu \mathrm{g}$

$\mathrm{ZnSO}_{4} \cdot 7 \mathrm{H}_{2} \mathrm{O}$

$400 \mu \mathrm{g}$

$\mathrm{FeCl}_{3} \cdot 6 \mathrm{H}_{2} \mathrm{O}$

$200 \mu \mathrm{g}$

$\mathrm{MnSO}_{4} \cdot \mathrm{H}_{2} \mathrm{O}$

$200 \mu \mathrm{g}$

$\mathrm{Na}_{2} \mathrm{MoO}_{4} \cdot 2 \mathrm{H}_{2} \mathrm{O}$

$200 \mu \mathrm{g}$

$\mathrm{KI}$

$100 \mu \mathrm{g}$

$\mathrm{CuSO}_{4} \cdot 5 \mathrm{H}_{2} \mathrm{O}$

$40 \mu \mathrm{g}$

Inositol

$2 \mathrm{mg}$

Pyridoxine $\cdot \mathrm{HCl}$

$400 \mu \mathrm{g}$

Thiamine $\cdot \mathrm{HCl}$

$400 \mu \mathrm{g}$

Biotin

$2 \mu \mathrm{g}$

Ca-pantothenate

$2.5 \mu \mathrm{g}$

Distilled water

to $1,000 \mathrm{ml}$ 


\section{RESULTS AND DISCUSSION}

Relationship between cell concentration and transmission value

In general, a range that gives a linear relation between the transmission value and the logarithm of cell concentration becomes narrow due to a multiscattering. The range is fixed characteristically by the structure of an apparatus and by the light path. The range given by this apparatus was determined by using yeast suspensions diluted at various concentrations. As shown in Fig. 5, linear relationship between the transmission value and the

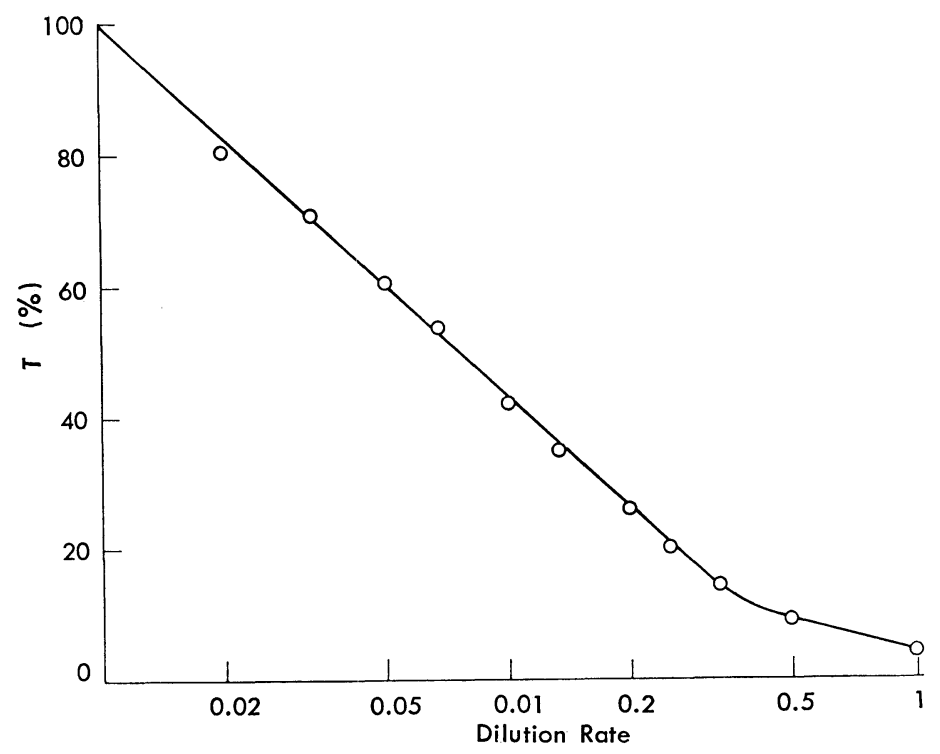

Fig. 5. Relationship between percentage transmission and cell concentration. Washed yeast suspensions diluted at various concentrations were used.

logarithm of cell density is obtained at the range of more than $15 \%$ transmission in this apparatus.

Effect of amount of media in L-tubes on growth

In this apparatus, at least $5 \mathrm{ml}$ of media are needed. This volume is necessary to fill up the L-tubes up to an upper side of a light-transmission hole. The effect of various amounts of medium (Medium B) contained in Ltubes on the growth of strictly aerobic $P$. alcaligenes was tested. As shown in Fig. 6-A, the maximum growth rate was obtained in $5 \mathrm{ml}$ of medium. Therefore, for the growth of aerobic microbes in the L-tubes it is desirable to employ the smallest volume $(5 \mathrm{ml})$ of medium. In some microbes which require a sufficient supply of oxygen, the use of a T-form tube may be re- 


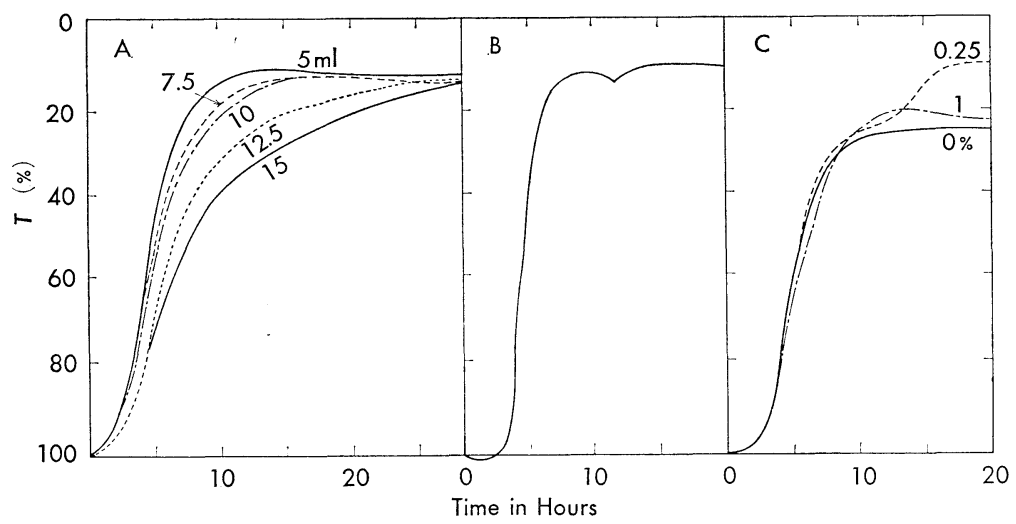

Fig. 6. Growth curves of various organisms. Pseudomonas alcaligenes grown in L-tubes containing various amounts of Medium B. B: Bacillus megaterium grown in Medium A. C: Escherichia coli grown in Medium B supplemented with various amounts of glucose.

Turbidity was measured 4 times an hour.

commendable to increase an air space in a culture vessel.

Several examples of growth curves

SLEPECKY and LAW (4) have reported on the sporulation of Barillus megaterium $\mathrm{KM}$ that a change in the optical density is characteristic showing a stepwise increase in the early stationary phase. Fig. 6-B shows a pronounced stepwise change during the course of sporulation in B. megaterium. In addition, when bacterial spores used as inoculum germinated in the medium, an appreciable decrease in the optical density was also recorded.

E. coli K 12 cultured in nutrient broth (Medium B) containing $0.25 \%$ glucose gave a growth curve showing a phenomenon of "diauxie" (Fig. 6C). By contrast, when Medium B was supplemented with $1 \%$ glucose, E. coli did not show such a diauxie type growth.

It was reported in the previous paper (1) that a variant strain of baker's yeast always appeared, when the yeast cells were incubated for 2 or more days in the pantothenate-deficient medium and that the variant strain thus obtained could grow well even at very low concentrations of pantothenate. The process of the appearance of such variant was followed using the pantothenatedeficient medium (Medium C) by plotting once an hour over 10 days (Fig. 7). Though some differences in growth curves were obtained from one tube to another probably because of the spontaneous nature of the mutation, the time lag of growth ranging from 2 to 3 days and a stepwise slow growth were observed. Thus, a "Bioscanner" was proved to be useful for such a long term experiment.

It should be mentioned that when we used baker's yeast, which is con- 


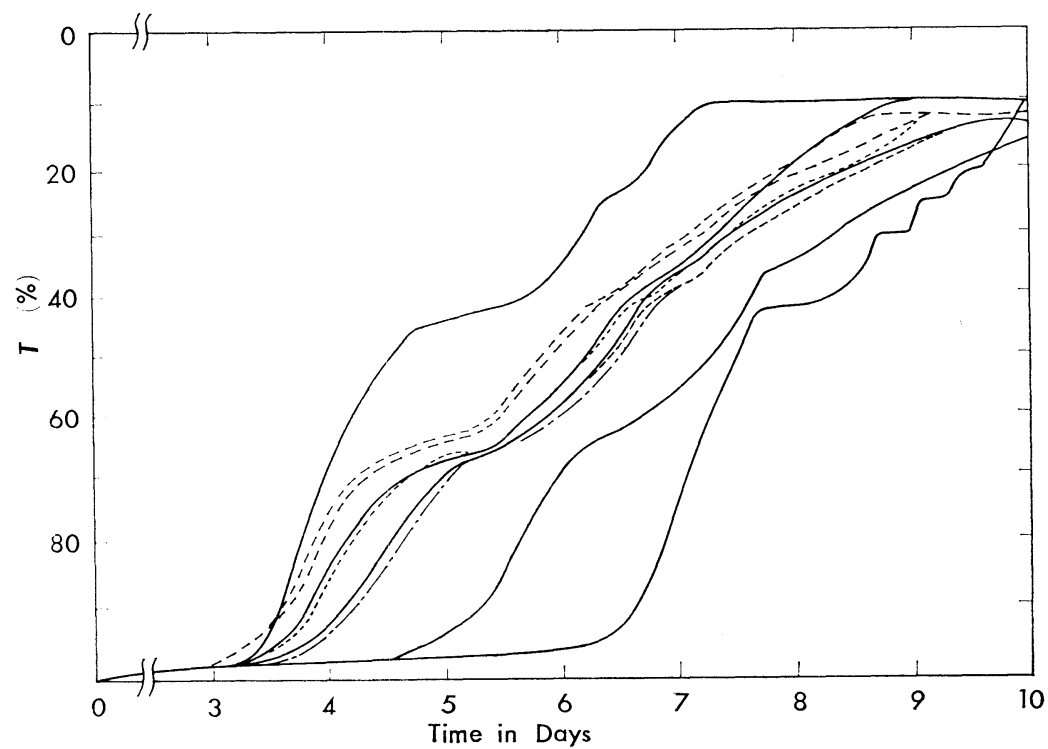

Fig. 7. Growth curves showing the appearance of variant strains of baker's yeast BA-1 being capable of growth even at very low concentrations of pantothenate.

Cells were grown in Medium C containing $2.5 \mu \mathrm{g}$ of pantothenate. Turbidity was measured once an hour.

sidered to be easily sedimentable, no disturbance in measuring turbidity was encountered even when L-tubes were kept unshaken for a $3.5 \mathrm{~min}$ period of measurement.

\section{Some additional comments}

This apparatus is less sensitive to color changes in media, though a glass filter $(660 \mathrm{~m} \mu)$ is employed. Therefore, the application of this apparatus on recording the course of color change is disadvantageous.

In this scanning system, a photocell returns to the original position only when 24 plottings are completed even when less than 21 samples are tested. However, it seems possible to shorten a scanning period without any essential reconstruction.

\section{REFERENCES}

1) H. Kuraishi: J. Gen. Appl. Microbiol., 12, 103 (1966).

2) H. H. Martin and J. W. Foster: J. Bacteriol., 76, 167 (1958).

3) T. Yamakawa, K. Aida and T. Uemura: J. Gen. Appl. Microbiol., 12, 337 (1966).

4) R. A. Slepecky and J. H. LAW: J. Bacteriol., 82, 37 (1961). 